
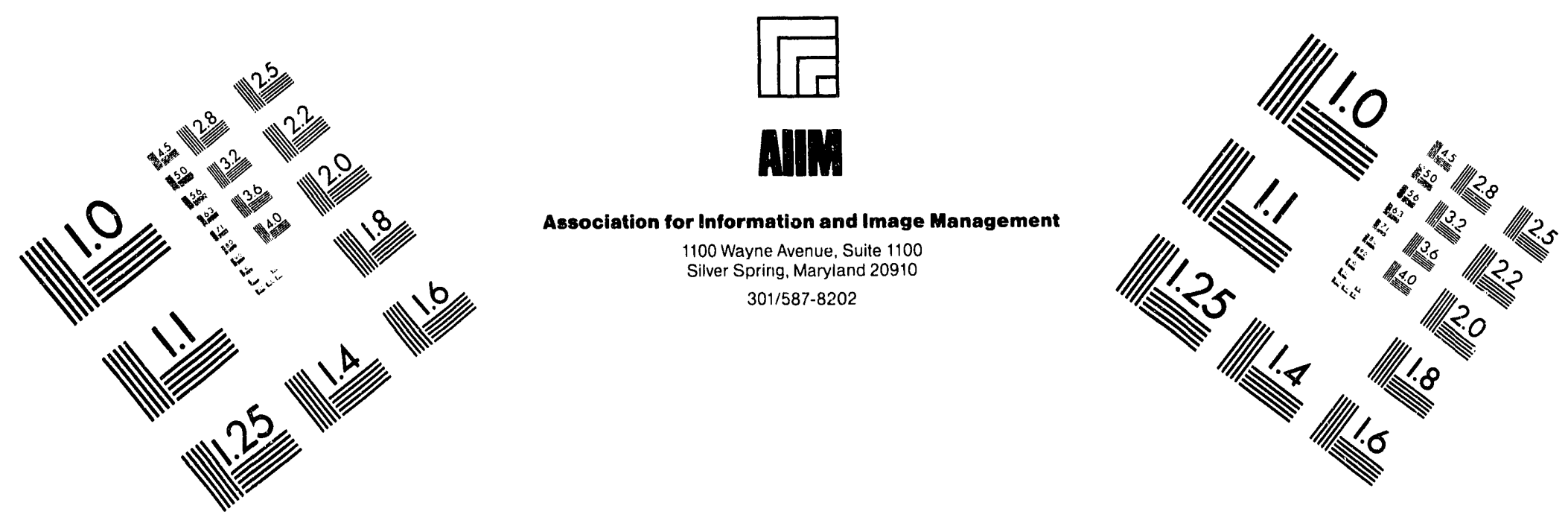

\title{
Centimeter
}

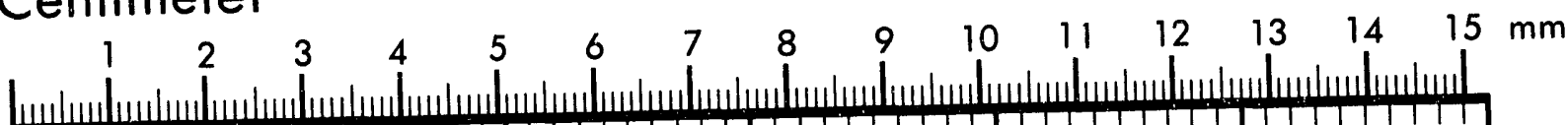

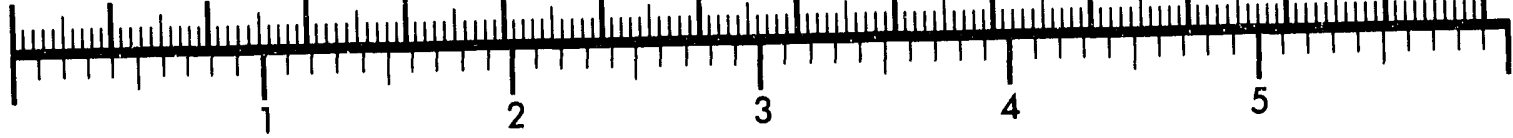
Inches
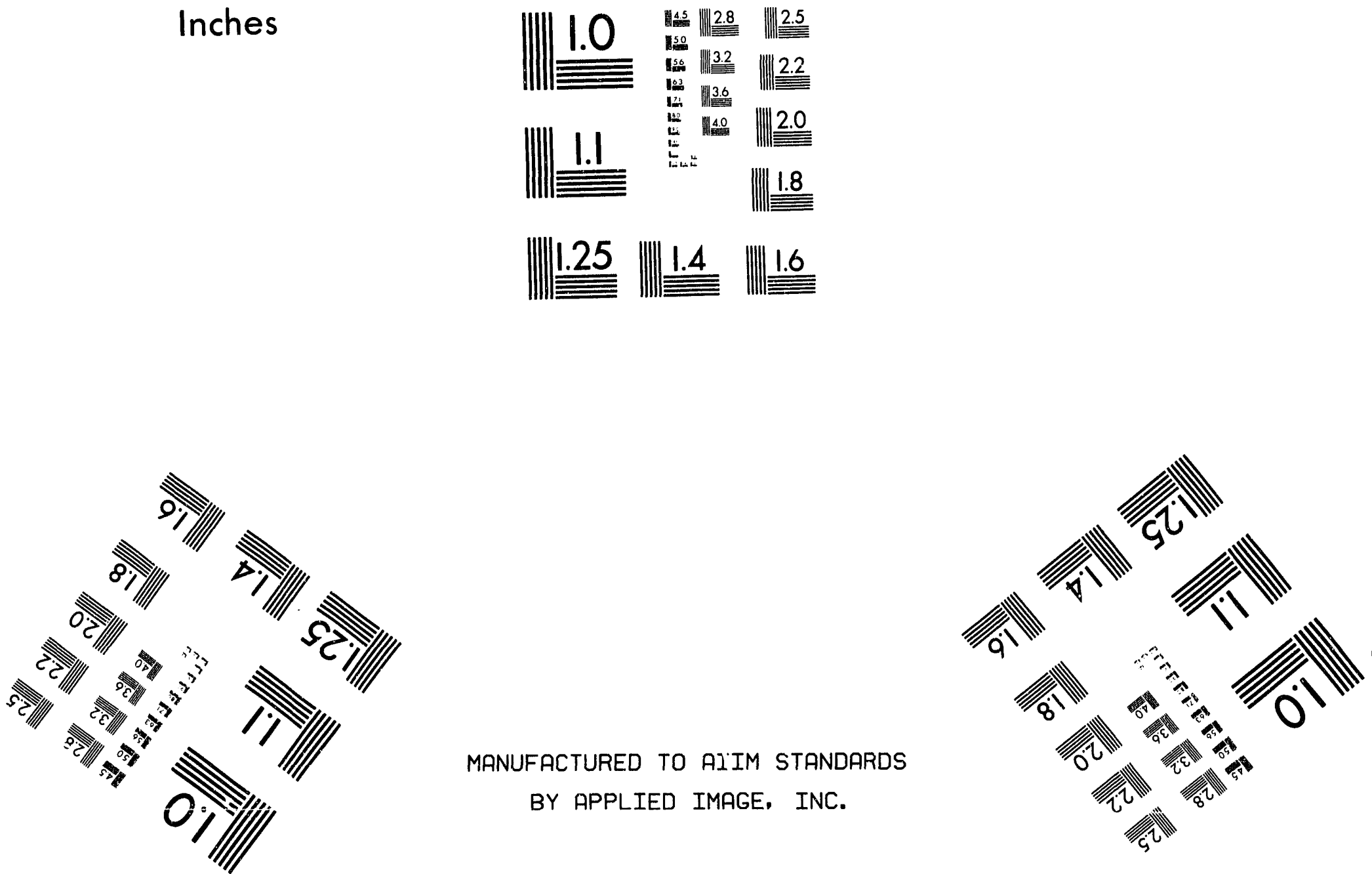

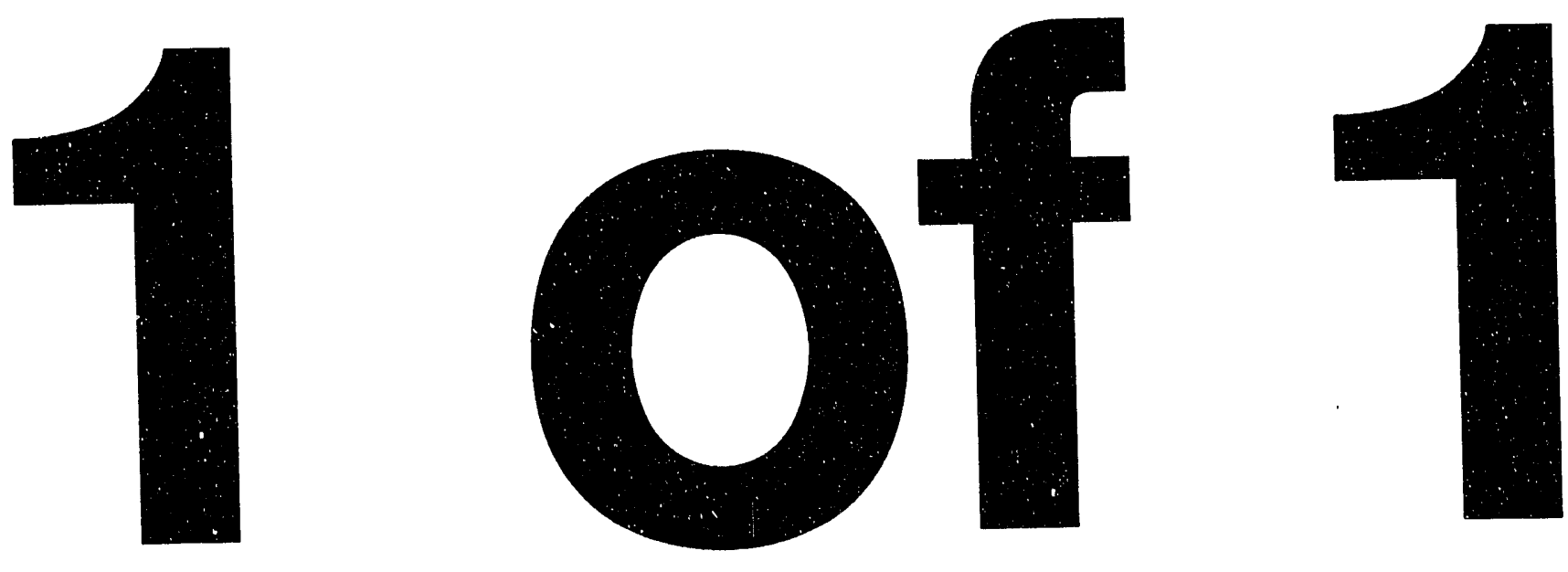


\title{
Cont-930367-6
}

\section{AFTER SEVEN YEARS, WHERE'S THE CONSENSUS?*}

M. R. Norman

Materials Science Division

Argonne National Laboratory

Argonne, IL 60439

\begin{abstract}
The submitted manuscript has been authored by a contractor of the U. S. Government under contract No. W-31-109-ENG-38.

Accordingly, the U.S. Government retains a nonexclusive, rnyalty-free license to publish or reproduce the published form of this contribution, or allow others to do so, for
\end{abstract} U.S. Government purposes.

APRIL 1993

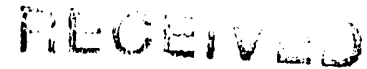

JUN $\{41933$

OSTI

/rlp

INVITED TALK: Submitted to Conference on Spectroscopies in Novel Superconductors, Santa Fe, NM, March 17-19, 1993.

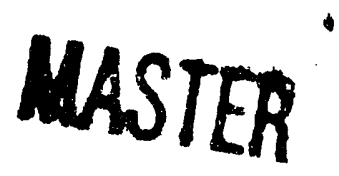

* Work Supported by the U.S. Department of Energy, BES-Materials Sciences, under Contract \#W-31-109-ENG-38. 


\title{
AFTER SEVEN YEARS, WHERE'S THE CONSENSUS?
}

\author{
M. R. NORMAN \\ Materials Science Division, Argonne National Lab, Argonne, IL 60439
}

\begin{abstract}
A summary of the theoretical part of the conference is given.
Keywords: theory, high temperature superconductors

It has been about seven years since the discovery of copper oxide superconductors. One might have thought that after such a long timc, some sort of consensus would have been reached in the field concerning the correct theory for this phenomenon. What was made quite apparent in the conference, though, is that no such consensus seems to be in sight.
\end{abstract}

Basically, theory can be divided into the following camps:

\begin{tabular}{ll} 
Revolutionary & Exotic \\
\hline $\begin{array}{l}\text { Luttinger liquid } \\
\text { spin-charge separation }\end{array}$ & AF spin fluctuations \\
gauge theories, anyons & nested Fermi liquid \\
marginal Fermi liquid & excitons, spin bags \\
odd frequency pairing
\end{tabular}

Qrdinary phonons

The ordinary camp is saying that the same old electron-phonon mechanism we have come to know and love over the past 40 years is happening in the cuprates, too. These people have taken quite a bit of abuse from their colleagues (after all, the Nobel prize allotment for this mechanism has become exhausted long ago, so who wants to work on such a boring thing as that), but they have had the grit to stick it out, and there is some evidence in their favor. After all, estimates of the electron-phonon coupling constant using standard methods indicate a $\lambda$ of about 1 , and this is consistent with some of the experimental data. This is sufficient to explain $T_{c}$ in the 214 system, as Pickett pointed out. If anything, though, these are underestimates, since the calculations assume perfect metallic screening. This assumption is particular!y bad for apical oxygens, where calculations which don't assume any screening yield enormously large values of $\lambda$ for these modes. Presumably, the truth lies somewhere in between. Of course, there are a lot of unusual properties associated with the cuprates that has led most people in the field to abandon an electron-phonon model. If so, then one has to understand why the electron-phonon interaction doesn't play any role despite the large value of $\lambda$, i.e., one has to find a way of putting the rabbit back into the hat, a difficult proposition for a Landau quasiparticle approach. In the end, though, what goes around, comes around, as even Phil Anderson was talking about an inplane electron-phonon model during his talk (the mandatory exotic ingredient being an enhancement of $\mathrm{T}_{c}$ by interplanar tunneling of electron pairs, a model which provoked quite a bit of lively discussion).

The exotic camp, meanwhile, simply replaces phonons by something else ons. A favorite these days are models based on antiferromagnetic spin fluctuations, a model which has had a lot of visibilicy in the field of heavy fermion superconductivity, where the electrons also exhibit strong correlations. These models exhibit an approximate linear temperature dependence of the resistivity above a low temperature scale $T^{*}$, below which they predict the canonical $\mathrm{T}^{2}$ behavior of Fermi liquid theory. $\mathrm{T}^{*}$ can be driven to zero by sitting on top of a vanHove singularity, or by being at the $T=0$ quantum critical point for a 2D antiferromagnet (the latter pointed out by Moriya's group). In the 3D regime, $\mathrm{T}^{1.5}$ behavior is predicted by Moriya's group, and such behavior has been inferred for overdoped 214 . Estimates of the superconducting transition temperature for these models vary quite a bit. Calculations by Levin's group give $T_{c}$ of around $10 \mathrm{~K}$, compared to the $100 \mathrm{~K}$ value calculated by Pines' and Moriya's groups (in all cases, $d_{x} 2-y^{2}$ pairing is found, in agreement with pioneering calculations of Scalapino for the 2D Hubbard model). The difference is due to two things. The Levin model is based on neutron scattering data, whereas that of Pines is based on NMR data. The inferred momentum 
dependence of the interaction is stronger in the NMR model (a point raised at this conference by Varma). Another problem is the appropriate estimate of the coupling constant to use in the gap equations. Technically, this I is the same I that is used to renormalize the non-interacting susceptibility in an RPA-based approach, but that constraint doesn't secm to be consistently implemented by any group (i.e., it is usually fit to give the right $T_{c}$ ). In the Levin case, this is fit to give the right slope for the resistivity. As this fit has been known for a long time to yield an upper bound to $\lambda_{t r}$ of around 0.4 (Gurvitch and Fiory), a low $T_{c}$ results. In fact, this low estimate for $\lambda_{t r}$ is a big problem for any theory. An additional problem pointed out by Batlogg is that there is no experimental correlation between the slope and $\mathrm{T}_{\mathrm{c}}$. Perhaps the best thing about this model is the prediction of only one symmetry for the gap function, $d_{x^{2}-y^{2}}$.

There is a big gulf between the revolutionary camp and the others. Although the others fight about a pairing mechanism, they all use some sort of Fermi liquid based approach. The revolutionaries, though, say that such an approach has to be patched up too badly to be of any real use for the cuprates. They propose to abandon this approach in favor of methods more appropriate for low dimensional models, where a linear resistivity automatir. ally comes out and persists to zero temperature (unless interrupted by the superconducting phase transition). This basically implies an abandonment of the Landau quasiparticle approach. Although in strongly correlated Fermi liquids, only a small part of the spectral weight sits in the quasiparticle pole, it is still the important piece of the physics. In the revolutionary models, though, there is no quasiparticle pole, and all the action is in the "incoherent" part of the Greens function. Although this camp has been rich in ideas, it suffers from the fact that all the machinery developed over the past 40 years to tackle electron correlations in Fermi liquids is largely inappropriate here. Thus, new techniques will have to be developed to be on the same computational footing as the other camps. An example of this was a drawing in Fihil Anderson's talk, where little arcs were drawn with the origin at the holon "Fermi surface" with the arcs centered at the spinon "Fermi surface". He attempted to connect this drawing with experimental photoemission results on BSCCO, but one must admit that there is a big gap between such a drawing and an actual calculation of photoemission linewidths. Progress on these matters is being made. As Varma pointed out, there are several model Hamiltonians which exist now that exhibit marginal Fermi liquid behavior, and experience with solving with these Hamiltonians will certainly accelerate our abilities to deal with systems where perturbing about a Fermi liquid fixed point is not appropriate. Another interesting point is that "marginal" behavior has been seen in several 3D magnets where the transition temperature has been forced to zero by pressure, and also has been seen in certain doped heavy fermion metals.

Still, it is somewhat disquieting to see not even a partial consensus after this amount of time. One can contrast this with heavy fermions, where although there is no overall agreement on the pairing mechanism, virtually all theories lie in the exotic camp.

An exciting issue brought up at the conference is the question of d-wave pairing in the cuprates. Evidence for this include a marked temperature dependence of the anisotropy of the NMR relaxation rate below $T_{c}$ by Slichter's group, a strong anisotropy of the superconducting gap function exhibited by angle resolved photoemission data in BSCCO (Spicer's group), a linear temperature dependence of the penetration depth seen by Hardy, and the famous $3 \Delta$ dip seen in both tunneling and photoemission as discussed by Coffey. The question of impurities will be addressed below. All of the above results merely reflect on the anisotropy of the gap function (with the exception of NMR, where coherence factors enter), so although they are consistent with a $d$-wave gap with nodes, they don't necessarily rule out a highly anisotropic gap with full rotational symmetry of the group. Various experiments have been suggested to directly couple to the phase of the order parameter. A Josephson junction experiment which can differentiate between s-wave and d-wave has been proposed by both Leggett and Sigrist and Rice, and the experiments are currently being performed by vanHarlingen at Urbana. Scalapino has suggested looking at what happens to the phonon linewidth as one goes through $T_{c}$ for large $q$ phonons where s- and d-wave coherence factors are dramatically different. Still, there is a lot of work to be done. To demonstrate this, we contrast what is known about this issue in heavy fermions with high $T_{c}$ 
Heavy Fermions

Knight shift

NMR and $\mu$ SR relaxation

transverse, longitudinal ultrasound

torsional oscillator

specific heat, thermal conductivity

$\mathrm{H}_{\mathrm{c} 2}$ and $\mathrm{H}_{\mathrm{c} 1}$ anomalies

penetration depth $(\omega=0$ and $\omega>0)$

coexistence of magnetism and SC

influence of impurities

Josephson and proximity effect
High $\mathrm{T}_{\mathrm{C}}$

NMR rēlaxation

angle resolved photoemission

penetration depth $(\omega=0)$

$3 \Delta$ dip in tunneling

Despite all the work done in heavy fermions, the actual symmetry of the gap function has still not been nailed down (not even the parity!). Still the question is a relatively straightforward one, and with the number of people involved with high $T_{c}$, it may well be sorted out in the next year or two.

An interesting thing left off the high $\Upsilon_{c}$ side of the above table were impurities. In heavy fermions, impurity effects are rather severe, i.e., if you grind $\mathrm{UPt}_{3}$, superconductivity disappears. This can be contrasted with high $\mathrm{T}_{\mathrm{c}}$ materials, where one must dope the material to obtain superconductivity, and in fact this material can be made by high school students. On the other hand, extrapolations of the resistivity to zero temperature for optimal doping give a very low value of the residual resistivity. Calculations by Levin's group indicate that for non-magnetic impurities in a d-wave state in YBCO, $T_{c}$ should disappear for residual resistivities of order $50 \mu \Omega \mathrm{cm}$. An interesting experiment reported on here by Kitaoka was on $\mathrm{Zn}$-doped $\mathrm{YBCO}$, where it was claimed that $\mathrm{Zn}$ went in non-magnetically (as opposed to previous experiments which indicated $\mathrm{Zn}$ went in magnetically), yet the $\mathrm{T}_{\mathrm{c}}$ suppression was even more rapid than with $\mathrm{Ni}$ doping. Evidence from NMR indicates that antiferromagnetic correlations are being suppressed by the $\mathrm{Zn}$, so this result is taken not only to be consistent with dwave pairing, but with $d$-wave pairing mediated by antiferromagnetic spin fluctuations (the result being backed up by calculations presented here by Monthoux). One problem, of course, is that it is well known that structural coherence of the $\mathrm{CuO}$ planes is needed for superconductivity, and little is known at this stage what the $\mathrm{Zn}$ is doing to the $\mathrm{CuO}$ planes (or, for that matter, how it influences the chains).

Another dangerous area is tunneling, where which results to believe and which not to believe varies depending on who you talk to. The results at this meeting seem to show a d-wave gap in BSCCO (Mihaly), but a "clean" gap in YBCO (Hasegawa).

I was asked by someone at the conference to say something nice about band theory. The best thing I can say is that band theory is like a prostitute. You get what you can out of her, then spurn her afterwards (a certain member of the band theory community asked me afterwards whether this made him a pimp). This is not meant to be a derogatory statement, but to highlight that the many-body community feels free to take parameters from a band calculation to use in their favorite model, but deride the calculation itself as "one-electron theory only pursued by simpletons" (thus, perhaps rape is a better term than prostitution). The fact is, it is somewhat amazing how ignorant some members of the many-body community are about density functional theory after 30 years time (not to be bigoted, certain band theory practitioners also fall in this category). The eigenvalue spectrum has to be taken with a very large grain of salt (after all, they merely act as Lagrange multipliers in the energy functional). Despite this, it is rather intriguing to see that such a complicated Fermi surface as predicted by band theory for YBCO seems to be confirmed by various experiments discussed here. Angle-resolved photoemission, as reported by Veal and Campuzano, sees the two barrel sheets (bonding and antibonding combinations of the $\mathrm{CuO}$ double layers), positrons, as reported by Manuel and Smedskjaer, see the ridge sheet coming from the chains, and several dHvA experiments (Los Alamos, Tohoku, and Grenoble groups) claim to see the small zone corner piece (another chain-related feature).

This brings us to the intere $\mathrm{g}$ question of c-axis dispersion, something discussed quite a bit by Phil 
Anderson, and also experimentally by Lance Cooper. Most c-axis measurements indicate non-metallic behavior (exceptions being overdoped 214 and optimally doped $Y$ BCO). Anderson feels that the normal state of high $\mathrm{T}_{c}$ exhibits incoherent behavior along the $c$-axis, and that coherence is restored in the superconducting state. This leads to a gain in free energy, and thus is responsible in his eyes for the large boost in $T_{c}$ seen in these materials (an idea backed up by recent calculations of Chakravarty). If this is so, it is difficult to understand why there is no correlation between $\rho_{c}$ and $T_{c}$ (as pointed out by Iye), or why the $c$-axis pressure derivative of $\mathrm{T}_{\mathrm{c}}$ is almost zero for $\mathrm{YBCO}_{7}$. Moreover, we can contrast the rather different behavior of $\mathrm{YBCO}_{7}$ and $\mathrm{O}_{6.7}$

$\begin{array}{lll} & \mathrm{O}_{7} & \mathrm{O}_{6.7} \\ \rho_{\mathrm{c}} & \mathrm{T} & 1 / \mathrm{T} \\ \sigma_{c}(\omega) & \text { Drude } & \text { non-Drude } \\ \mathrm{dT} \mathrm{T}_{\mathrm{d}} \mathrm{dP} & -0 & \text { negative }\end{array}$

These behaviors are quite different, yet the $\mathrm{T}_{c}$ of $\mathrm{O}_{7}$ is only about $50 \%$ higher than $\mathrm{O}_{6.7}$. Therefore, at this stage, it is unclear what role c-axis coupling, if any, plays in the superconductivity. Altemate models to Anderson/Chakravarty have appeared, including the influence of in-plane fluctuations on caxis transport (Leggett), the tortuous path model of Welp and Crabtree, and two models which attribute the uptum of $\rho_{c}$ above $T_{c}$ to superconducting fluctuations (Gray-Kim, Ioffe-Larkin-Varlamov-Yu). Another way of getting incoherent behavior along the $c$ axis from microscopic theory is to consider that for metallic systems, the exchange-correlation (XC) hole of the electron is tied to the electron, but for insulators, the XC hole can move away (this is responsible for the Mott gap in density functional theory). Thus, for transport in the plane, the $\mathrm{XC}$ hole is tied to the electron due to metallic screening, but for transport between planes, the XC hole might be left behind, only to rejoin the electron whon it reaches the next plane, possibly leading to "insulating" behavior for c-axis transport.

Finally, we mention the various interesting $\mathrm{dHvA}$ results talked about at the conference. Several experiments have been done on trying to measure dHvA oscillations in the mixed (type II) phase:

$\mathrm{NbSe}_{2}$ (Graebner-Robbins, Onuki, Springford); $\mathrm{Nb}$ (Aoki); $\mathrm{Nb}_{3} \mathrm{Sn}$ (Springford); $\mathrm{V}_{3} \mathrm{Si}$ (Mueller-ArkoLowndes); YBCO (Los Alamos, Sendai, Grenoble); T1-2201 (Sendai); BKBO (Goodrich-Los Alamos)

The last results on BKBO are brand new (they were produced the same day that Goodrich talked about them) and were quite impressive, showing beautiful, large magneto-oscillations below $\mathrm{H}_{c 2}$ with a frequency in agreement with band calculations of Koelling (which shows only one large sheet for the Fermi surface). Some experiments, though, haven't seen rnagneto-oscillations at all below $\mathrm{H}_{\mathrm{c} 2}(\mathrm{Nb}$, $\mathrm{Nb}_{3} \mathrm{Sn}$ ). The theory behind this has been developed by Stephen and Maki, the basic idea is that for strong type-II superconductors, the field penetrates fairly homogeneously, thus allowing magnetooscillations to be seen $(\mathrm{Nb}$ is a weak type-II material, which is what is killing the oscillations in that case). What does happen, though, is that the Landau levels get broadened out into bands due to pairing correlations, thus the effective Dingle (scattering) temperature increases in the mixed phase, leading to a change in slope when the logarithm of the amplitude is plotted versus $1 / \mathrm{H}$. The change in slope is predicted to be quite large for $\mathrm{Nb}_{3} \mathrm{Sn}$ which is presumably why no magneto-oscillations are seen in that case (Springford, private communication). Obviously, these calculations need to be repeated for the other systems (calculations have been done for $\mathrm{NbSe}_{2}$, the fit indicating suppression of the superconducting gap for the small pocket of the Fermi surface seen, consistent with the fact that this pocket is most likely caused by a CDW distortion, with the two order parameters competing against each other on this pocket). As pointed by Maniv, an additional effect is possible in that the order parameter itself has magneto-oscillations. Although the period is the same as the "normal" magnetooscillations, the field dependence of the amplitude is different, leading to an unusual field dependence of the overall amplitude which has a maximum below $\mathrm{H}_{c 2}$. Haanappel gave some evidence at this meeting from the Grenoble data on YBCO that support this picture. At this stage, though, no theory exists which self-consistently includes the supercurrent response. A number of people are working on this, though, so hopefully by the next meeting, a more complete theory will exist. 
Work supported by the U. S. Dept. of Energy, Basic Energy Sciences, under Contract No. W-31-109ENG-38.

\section{DISCLAIMER}

This report was prepared as an account of work sponsored by an agency of the United States Government. Neither the United States Government nor any agency thereof, nor any of their employees, makes any warranty, express or implied, or assumes any legal liability or responsibility for the accuracy, completeness, or usefulness of any information, apparatus, product, or process disclosed, or represents that its use would not infringe privately owned rights. Reference herein to any specific commercial product, process, or service by trade name, trademark, manufacturer, or otherwise does not necessarily constitute or imply its endorsement, recommendation, or favoring by the United States Government or any agency thereof. The views and opinions of authors expressed herein do not necessarily state or reflect those of the United States Government or any agency thereof. 

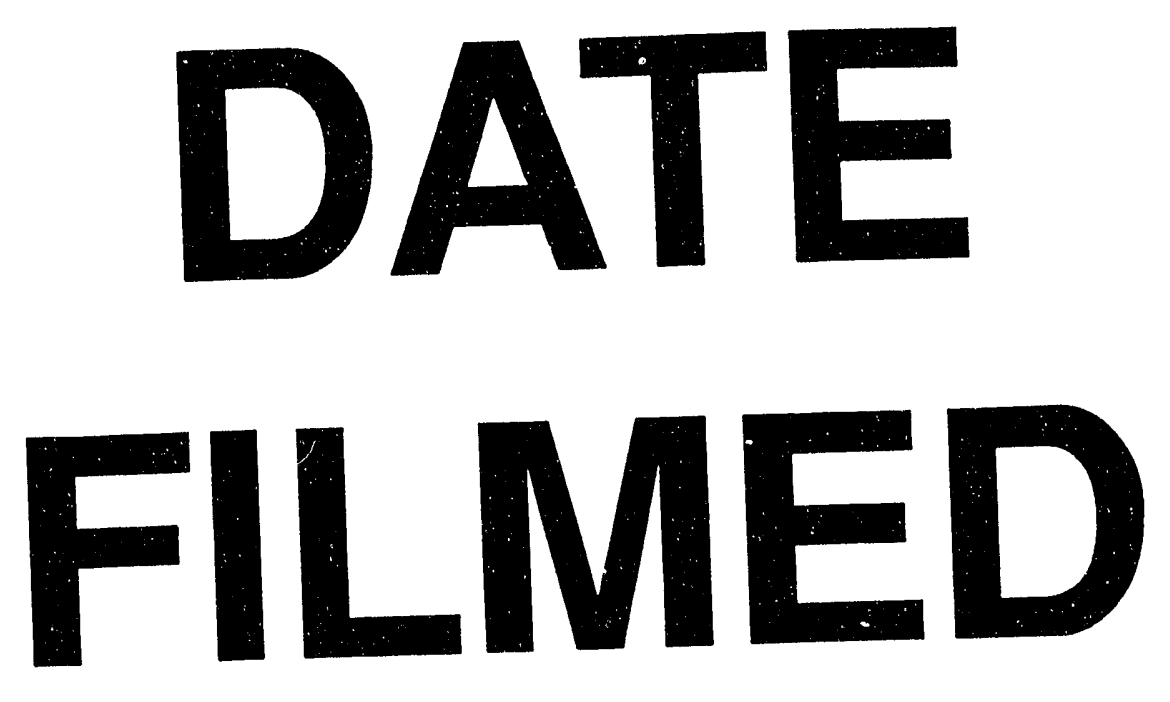

$$
7 / 29 / 93
$$
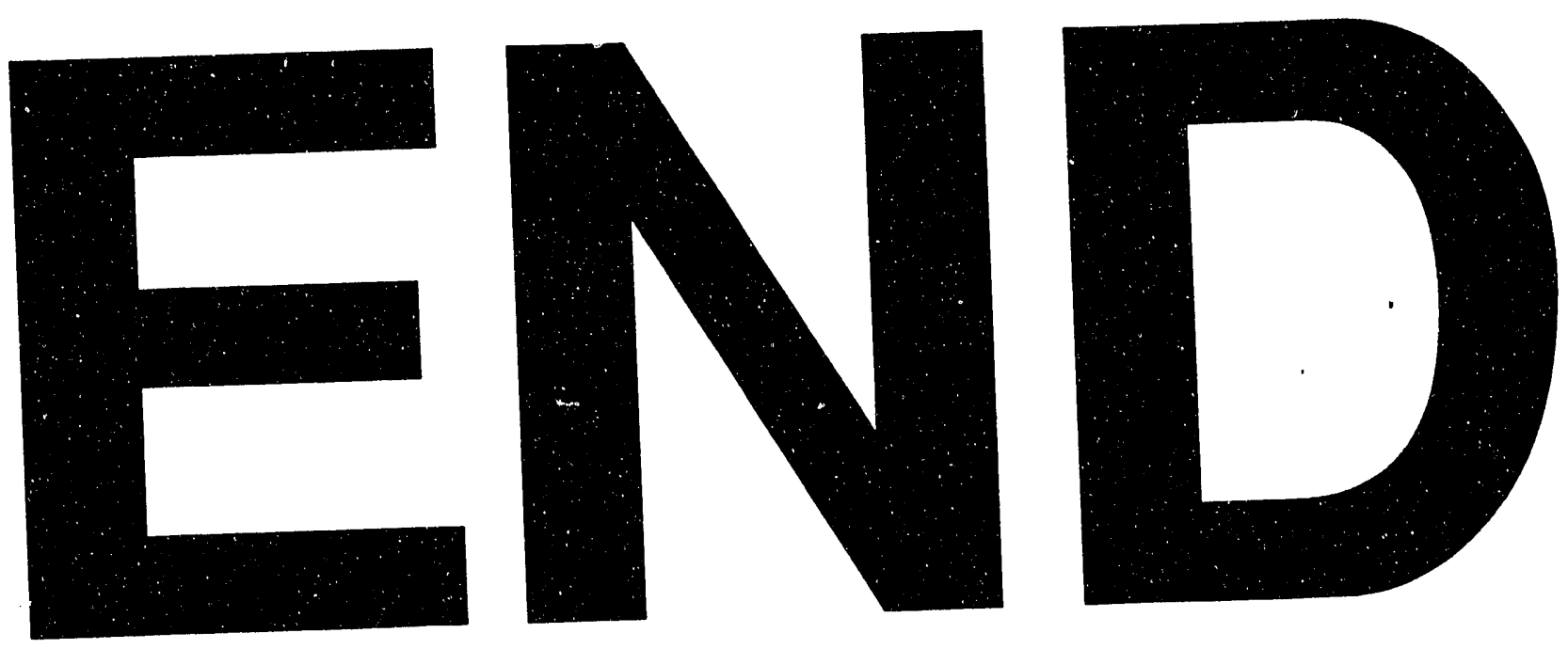
